\title{
Time-dependent density functional theory scheme for efficient calculations of dynamic (hyper)polarizabilities
}

\author{
Xavier Andrade ${ }^{\text {a) }}$ \\ Departamento de Física de Materiales, Facultad de Ciencias Químicas, UPV/EHU Centro Mixto \\ CSIC-UPV/EHU and European Theoretical Spectroscopy Facility (ETSF), E-20018 San Sebastián, \\ Spain; Laboratoire des Solides Irradiés, CNRS-CEA-École Polytechnique, F-91128 Palaiseau, France
}

\author{
Silvana Botti \\ Laboratoire des Solides Irradiés, CNRS-CEA-École Polytechnique and European Theoretical Spectroscopy \\ Facility (ETSF), F-91128, France; and Centro de Física Computacional, Departamento de Física, \\ Universidade de Coimbra, P-3004516 Coimbra, Portugal \\ Miguel A. L. Marques \\ Centro de Física Computacional, Departamento de Física, Universidade de Coimbra, P-3004516 Coimbra, \\ Portugal; and European Theoretical Spectroscopy Facility (ETSF), E-20018 San Sebastián, Spain \\ Angel Rubio \\ Departamento de Física de Materiales, Facultad de Ciencias Químicas, UPV/EHU, Centro Mixto \\ CSIC-UPV/EHU, Donostia International Physics Center and European Theoretical Spectroscopy \\ Facility (ETSF), E-20018, San Sebastián, Spain
}

(Received 22 January 2007; accepted 29 March 2007; published online 10 May 2007)

\begin{abstract}
The authors present an efficient perturbative method to obtain both static and dynamic polarizabilities and hyperpolarizabilities of complex electronic systems. This approach is based on the solution of a frequency-dependent Sternheimer equation, within the formalism of time-dependent density functional theory, and allows the calculation of the response both in resonance and out of resonance. Furthermore, the excellent scaling with the number of atoms opens the way to the investigation of response properties of very large molecular systems. To demonstrate the capabilities of this method, they implemented it in a real-space (basis-set-free) code and applied it to benchmark molecules, namely, $\mathrm{CO}, \mathrm{H}_{2} \mathrm{O}$, and para-nitroaniline. Their results are in agreement with experimental and previous theoretical studies and fully validate their approach. (C) 2007 American Institute of Physics. [DOI: 10.1063/1.2733666]
\end{abstract}

\section{INTRODUCTION}

The optical properties of a material are essentially determined by the response of the electrons to an external field. If this applied field is small, the induced dipole of the system can be expanded in powers of the field. ${ }^{1,2}$ The first order coefficient is the so-called electric polarizability $\alpha$. This quantity describes, e.g., the dielectric properties of the material and how light is absorbed and emitted. In second order we obtain the first hyperpolarizability $\beta$ that is responsible for the processes of second harmonic generation, optical rectification, and Pockles effect. ${ }^{1}$ Higher order terms can be related to other electro-optical effects such as third harmonic generation, the Kerr effect, etc. Finally, polarizabilities are called static or dynamic if the perturbing field is static or frequency dependent.

The importance of the linear term, $\alpha$, is well known in physics and chemistry. ${ }^{3}$ On the other hand, nonlinear (i.e., beyond first order) optical effects have gained quite some interest lately due to their technological applications in optoelectronic devices. Nonlinear optical materials can be used to convert light to shorter (bluer) wavelengths, which can be focused to a smaller spot size. Shorter wavelength

${ }^{a)}$ Electronic mail: xavier@tddft.org light sources would hence yield higher density optical recording media (such as digital versatile disks and compact disks). Other applications include tunable light sources, image recognition systems, and adaptive optics.

Several methods have been used to calculate (hyper)polarizabilities of finite systems. ${ }^{4-8}$ In the static case, the simplest approach is finite differences ${ }^{9}$ that use the definition of the polarizabilities as derivatives of the dipole (or of the total energy) with respect to the applied field. Calculations are performed at various (small) field strengths, and the required derivatives are evaluated numerically. This method is simple and straightforward to implement. However, it requires many total energy evaluations, and these need to have a very high precision to obtain reasonable numerical derivatives. Moreover, it is not possible to generalize this idea to the dynamic case.

Another widely used approach is the perturbation theory, of which more than one flavor exists. In the sum-over-states method $^{4,10}$ the (hyper)polarizabilities are written as an infinite sum over occupied and empty states, which involves the ground state eigenvalues and dipole matrix elements. In a similar vein, one can obtain the polarizabilities from the corresponding response functions written in the product basis of occupied and empty states or in terms of Green's functions. ${ }^{11}$ Note that these techniques can be used for both static and 
dynamic responses. Although widely used by the community, these methods have several shortcomings. First, results are often difficult to converge with the number (and quality) of the empty states. Second, the scaling with the number of atoms is quite unfavorable, making the application to the study of large system hard, such as nanostructures or molecules with biological interest.

A different technique also used to obtain both static and dynamic linear polarizabilities is the direct solution of the time-dependent Schrödinger equation in real time. ${ }^{12}$ In this way only the occupied subspace is needed and the scaling with system size is excellent $\left[\mathcal{O}\left(N^{2}\right)\right.$, where $N$ is the number of atoms]. However, this method cannot be easily generalized to extract hyperpolarizabilities.

A very interesting approach that essentially solves the problems mentioned above is the Sternheimer equation. ${ }^{13} \mathrm{Al}-$ though a perturbative technique, it avoids the use of empty states and has a quite good scaling with the number of atoms. This method has already been used for the calculation of many response properties ${ }^{7}$ such as atomic vibrations (phonons), electron-phonon coupling, magnetic response, etc. In the domain of optical response, this method has been mainly used for static response, although a few first principles calculations for low frequency (far from resonance) (hyper)polarizabilities have appeared. ${ }^{14-17}$ Recently, a reformulation of the Sternheimer equation in a superoperator formalism was presented. ${ }^{18}$ When combined with a Lanczos solver, it allows us to calculate very efficiently the first order polarizability for the whole frequency spectrum. However, the generalization of this method to higher orders is not straightforward.

In this article, we propose a modified version of the Sternheimer equation that is able to cope with both static and dynamic responses in and out of resonance. The solution of the first order Sternheimer equation gives us access to both $\alpha$ and $\beta$. Higher order polarizabilities can be obtained from an hierarchy of Sternheimer equations. Exchange and correlation effects are treated at the level of density functional theory ${ }^{19}$ (DFT) for static polarizabilities and time-dependent DFT (TDDFT) (Ref. 20) for the dynamic case. Compared to other quantum-chemistry approaches, density functional methods have a somewhat lower accuracy but are lighter numerically, allowing the study of much larger systems. In the present work we focus on finite systems, but the method has also been applied to periodic systems for the nonresonant case. $^{21}$ Note that in the field of DFT, the Sternheimer equation is often referred to as the density functional perturbation theory. ${ }^{7}$

The rest of this article is organized as follows. In Sec. II we present the derivation of the frequency-dependent Sternheimer equation and show how to obtain the linear polarizability and first hyperpolarizability from its solution. In the following section we give some details concerning the implementation of our method. In Sec. IV we apply this theory to several test molecules, comparing our results to other calculations and experiments. Finally we present our conclusions and a brief outlook.

\section{THEORY}

\section{A. Linear response}

Within TDDFT, the quantum state of an interacting electronic system is described by the time-dependent KohnSham equations (atomic units will be used unless explicitly stated),

$$
i \frac{\partial}{\partial t} \psi_{m}(\boldsymbol{r}, t)=H_{\mathrm{KS}}(t) \psi_{m}(\boldsymbol{r}, t)
$$

The Kohn-Sham Hamiltonian is written as

$$
H_{\mathrm{KS}}=-\frac{\nabla^{2}}{2}+v_{\mathrm{ext}}(\boldsymbol{r}, t)+v_{\text {Hartree }}(\boldsymbol{r}, t)+v_{\mathrm{xc}}(\boldsymbol{r}, t),
$$

where the first term corresponds to the kinetic energy, and the following ones represent the external potential, the Hartree potential that describes the classical interaction between the electrons, and the exchange-correlation term that accounts for all nontrivial parts of the electron-electron interaction. Note that the Hartree and exchange-correlation terms are time dependent as they are functionals of the (timedependent) density. This latter quantity can be evaluated from the occupied Kohn-Sham orbitals,

$$
n(\boldsymbol{r}, t)=\sum_{m}^{\text {occ }}\left|\psi_{m}(\boldsymbol{r}, t)\right|^{2}
$$

We are concerned with external potentials that are the sum of a time-independent part, typically created by a set of nuclei, and a monochromatic electric field $v_{\text {field }}(\boldsymbol{r}, t)$ $=\sum_{i=1}^{3} \lambda_{i} r_{i} \cos (\omega t)$. If we assume that the magnitude of $\lambda$ is small, we can use the perturbation theory to expand the Kohn-Sham wave functions in powers of $\lambda$. The first order term reads

$$
\begin{aligned}
\psi_{m}(\boldsymbol{r}, t)= & e^{-i\left(\epsilon_{m}+\Sigma_{i=1}^{3} \lambda_{i} \epsilon_{m, i}^{(1)}\right) t}\left\{\psi_{m}^{(0)}(\boldsymbol{r})+\frac{1}{2} \sum_{i=1}^{3}\left[\lambda_{i} e^{i \omega t} \psi_{m, i}^{(1)}(\boldsymbol{r}, \omega)\right.\right. \\
& \left.+\lambda_{i} e^{-i \omega t} \psi_{m, i}^{(1)}(\boldsymbol{r},-\omega)\right]
\end{aligned},
$$

where $\psi_{m}^{(0)}(\boldsymbol{r})$ are the wave functions of the static Kohn-Sham Hamiltonian $H^{(0)}$ obtained by taking $\lambda=0$,

$$
H^{(0)} \psi_{m}^{(0)}(\boldsymbol{r})=\epsilon_{m} \psi_{m}^{(0)}(\boldsymbol{r})
$$

and $\psi_{m, i}^{(1)}(\boldsymbol{r}, \omega)$ are the first order variations of the timedependent Kohn-Sham wave functions.

From Eq. (4) and the definition of the time-dependent density [Eq. (3)], we can obtain the time-dependent density

$$
\begin{aligned}
n(\boldsymbol{r}, t)= & n^{(0)}(\boldsymbol{r})+\frac{1}{2} \sum_{i=1}^{3}\left[\lambda_{i} e^{i \omega t} n_{i}^{(1)}(\boldsymbol{r}, \omega)\right. \\
& \left.+\lambda_{i} e^{-i \omega t} n_{i}^{(1)}(\boldsymbol{r},-\omega)\right],
\end{aligned}
$$

with the following definition of the first order variation of the density: 


$$
\begin{aligned}
n_{i}^{(1)}(\boldsymbol{r}, \omega)= & \sum_{m}^{\mathrm{occ}}\left\{\left[\psi_{m}^{(0)}(\boldsymbol{r})\right]^{*} \psi_{m, i}^{(1)}(\boldsymbol{r}, \omega)\right. \\
& \left.+\left[\psi_{m, i}^{(1)}(\boldsymbol{r},-\omega)\right]^{*} \psi_{m}^{(0)}(\boldsymbol{r})\right\} .
\end{aligned}
$$

By replacing the expansion of the wave functions Eq. (4) in the time-dependent Kohn-Sham equation [Eq. (1)] and picking up the first order terms in $\lambda$, we arrive at a Sternheimer equation for the variations of the wave functions,

$$
\left\{H^{(0)}-\epsilon_{m} \pm \omega+i \eta\right\} \psi_{m, i}^{(1)}(\boldsymbol{r}, \pm \omega)=-P_{c} H_{i}^{(1)}( \pm \omega) \psi_{m}^{(0)}(\boldsymbol{r}),
$$

with the first order variation of the Kohn-Sham Hamiltonian as

$$
\begin{aligned}
H_{i}^{(1)}(\omega)= & r_{i}+\int \mathrm{d}^{3} r^{\prime} \frac{n^{(1)}\left(\boldsymbol{r}^{\prime}, \omega\right)}{\left|\boldsymbol{r}-\boldsymbol{r}^{\prime}\right|}+\int \mathrm{d}^{3} r^{\prime} f_{\mathrm{xc}}\left(\boldsymbol{r}, \boldsymbol{r}^{\prime}\right) n^{(1)} \\
& \times\left(\boldsymbol{r}^{\prime}, \omega\right),
\end{aligned}
$$

where $P_{c}$ is the projector onto the unoccupied subspace. The effect of this projector is to make the components of $\psi_{m, i}^{(1)}$ $\times(\boldsymbol{r}, \pm \omega)$ zero in the subspace of the occupied ground state wave functions. In linear response, these components do not contribute to the variation of the density; ${ }^{60}$ therefore, we can safely ignore the projector. This is important for large systems as the cost of the calculation of the projections scales quadratically with the number of orbitals.

The first term of $H_{i}^{(1)}(\omega)$ comes from the external perturbative field, while the next two represent the variation of the Hartree and exchange-correlation potentials. The exchangecorrelation kernel is a functional of the ground state density $n^{(0)}$ and is given by the functional derivative,

$$
f_{\mathrm{xc}}\left[n^{(0)}\right]\left(\boldsymbol{r}, \boldsymbol{r}^{\prime}\right)=\left.\frac{\delta v_{\mathrm{xc}}(\boldsymbol{r})}{\delta n\left(\boldsymbol{r}^{\prime}\right)}\right|_{n=n^{(0)}} .
$$

In the previous equations we made use of the adiabatic approximation to write $f_{\mathrm{xc}}$ as a frequency-independent quantity. Equations (7) and (8) form a set of self-consistent equations for linear response that only depend on the occupied ground state orbitals.

Note that we included in Eq. (8) a positive infinitesimal $\eta$. This term is essential to obtain the correct position of the poles of the causal response function and, therefore, to obtain the imaginary part of the polarizability. Furthermore, using a small, but finite, $\eta$ allows us to solve numerically the Sternheimer equation close to resonances, as it removes the divergences of this equation.

By following the same kind of reasoning, we can arrive at a hierarchy of Sternheimer equations for the higher order terms in $\lambda$. This will be needed for the calculation of $\gamma$, the second order hyperpolarizability, or higher order hyperpolarizabilities. ${ }^{22}$

\section{B. Polarizability}

The time-dependent dipole moment is defined as

$$
\mu_{i}(t)=\int \mathrm{d}^{3} r n(\boldsymbol{r}, t) r_{i}
$$

The polarizabilities are defined by the expansion of the dipole moment in terms of the electric field,

$$
\begin{aligned}
\mu_{i}(t)= & \mu_{i}(0)+\sum_{j}^{3} \alpha_{i j}\left(\omega_{j}\right) \lambda_{j} \cos \left(\omega_{j} t\right)+\frac{1}{2 !} \sum_{j, k=1}^{3} \lambda_{j} \lambda_{k} \\
& \times \beta_{i j k}\left(-\omega_{j}-\omega_{k} ; \omega_{j}, \omega_{k}\right) \cos \left(\omega_{j} t\right) \cos \left(\omega_{k} t\right)+\cdots .
\end{aligned}
$$

We must notice that there are several conventions for the definition of the (hyper)polarizabilities, which are conveniently detailed in Ref. 23. In this work we follow convention $A B$ [where the prefactors $1 / n$ ! are explicitly included in Eq. (12)] that appears to be the most used by the theoretical community. All referenced values have been converted to this convention.

If we replace expression (7) in Eq. (11) and compare with Eq. (12), we can obtain a formula for the polarizability in terms of the variation of the density,

$$
\alpha_{i j}(\omega)=\int \mathrm{d}^{3} r n_{j}^{(1)}(\boldsymbol{r}, \omega) r_{i} .
$$

The quantity most easily accessible experimentally is the photoabsorption cross section that can be evaluated directly from the linear polarizability,

$$
\bar{\sigma}(\omega)=\frac{4 \pi \omega}{c} \mathfrak{I m} \bar{\alpha}(\omega),
$$

where $\bar{\alpha}$ is the trace of the polarizability tensor,

$$
\bar{\alpha}(\omega)=\frac{1}{3} \sum_{i=1}^{3} \alpha_{i i}(\omega)
$$

\section{First hyperpolarizability}

If for the dynamic hyperpolarizabilities we follow the same procedure as before, we get an expression in terms of the second order variation of the density that requires the evaluation of higher order variations of the wave functions. However, it is possible to get the first hyperpolarizability directly from the first order variations by means of the $2 n$ +1 theorem. This theorem states that the $n$th order variations of the wave functions are enough to obtain the $2 n+1$ derivative of the energy. ${ }^{7,24}$ This theorem can be expanded to the dynamic case and allows us to write the first hyperpolarizability $\beta$ in terms of the first order variations of the wave functions. After some algebra, we arrive $\mathrm{at}^{21}$ 


$$
\begin{aligned}
\beta_{i j k}\left(-\omega_{1} ; \omega_{2}, \omega_{3}\right)= & -4 \sum_{P} \sum_{\zeta= \pm 1}\left\{\sum_{m}^{\mathrm{occ}} \int \mathrm{d}^{3} r \psi_{m, i}^{(1) *}\left(\boldsymbol{r},-\zeta \omega_{1}\right) H_{j}^{(1)}\left(\zeta \omega_{2}\right) \psi_{m, k}^{(1)}\left(\boldsymbol{r}, \zeta \omega_{3}\right)-\sum_{m n}^{\mathrm{occ}} \int \mathrm{d}^{3} r \psi_{m}^{(0)^{*}}(\boldsymbol{r}) H_{j}^{(1)}\left(\zeta \omega_{2}\right) \psi_{n}^{(0)}(\boldsymbol{r})\right. \\
& \left.\times \int \mathrm{d}^{3} r \psi_{n, i}^{(1) *}\left(\boldsymbol{r},-\zeta \omega_{1}\right) \psi_{m, k}^{(1)}\left(\boldsymbol{r}, \zeta \omega_{3}\right)-\frac{2}{3} \int \mathrm{d}^{3} r \int \mathrm{d}^{3} r^{\prime} \int \mathrm{d}^{3} r^{\prime \prime} K_{\mathrm{xc}}\left(\boldsymbol{r}, \boldsymbol{r}^{\prime}, \boldsymbol{r}^{\prime \prime}\right) n_{i}^{(1)}\left(\boldsymbol{r}, \omega_{1}\right) n_{j}^{(1)}\left(\boldsymbol{r}^{\prime}, \omega_{2}\right) n_{k}^{(1)}\left(\boldsymbol{r}^{\prime \prime}, \omega_{3}\right)\right\},
\end{aligned}
$$

where the first sum is over the permutations $P$ of the pairs $\left(i,-\omega_{1}\right),\left(j, \omega_{2}\right)$, and $\left(k, \omega_{3}\right)$ and the exchange-correlation kernel, written in the adiabatic approximation, reads

$$
K_{\mathrm{xc}}\left(\boldsymbol{r}, \boldsymbol{r}^{\prime}, \boldsymbol{r}^{\prime \prime}\right)=\left.\frac{\delta^{2} v_{\mathrm{xc}}(\boldsymbol{r})}{\delta n\left(\boldsymbol{r}^{\prime}\right) \delta n\left(\boldsymbol{r}^{\prime \prime}\right)}\right|_{n=n^{(0)}} .
$$

The first hyperpolarizability tensor has 27 components and is in general nonsymmetric. The quantity that is experimentally relevant is

$$
\beta_{\|}=\frac{1}{5} \sum_{i=1}^{3}\left(\beta_{z i i}+\beta_{i z i}+\beta_{i i z}\right),
$$

where $z$ is oriented in the direction of the dipole moment of molecule. Sometimes the equivalent quantity $\beta_{\mathrm{vec}}=\beta_{z}$ $=(5 / 3) \beta_{\|}$is used.

\section{IMPLEMENTATION}

This scheme has been implemented using a real-space grid based formulation in the code octopus. ${ }^{25}$ We have chosen a real-space grid as it allows us a systematic convergence of the results (hyperpolarizabilities are notoriously difficult to converge with localized basis sets). However, uniform grids cannot easily describe all-electron atoms, so we replace the electron-nuclear Coulomb interaction by KleinmanBylander pseudopotentials. This is, however, a well controlled approximation for the systems we are interested in.

In (TD)DFT several approximations exist for the exchange-correlation term. ${ }^{26}$ In our approach we can treat the exchange-correlation term at two levels; one is the ground state exchange-correlation potential involved in the calculation of the ground state wave functions and the other is the exchange-correlation kernel. For the ground state, except were noted otherwise, we use the local density approximation (LDA); we will also use the exact exchange functional in the Krieger-Li-Iafrate ${ }^{32}$ (KLI) approximation. For the exchange-correlation kernel, we have decided to use, due to its simplicity, the adiabatic local density approximation (ALDA) (although our scheme is quite general and can, in principle, be applied to any exchange-correlation functional).

The LDA is a well studied approximation and is quite reliable in the prediction of many properties. One important main defect in this context is the wrong asymptotic part of the LDA exchange-correlation potential that for neutral systems decays exponentially instead of falling as $1 / r$. This usually leads to small highest-occupied-molecular-orbitallowest-unoccupied-molecular-orbital gaps, which implies systems that are too polarizable, in contrast to Hartree-Fock where the gap is larger and the magnitudes of the polarizabilities are underestimated. The exchange and correlation kernel will contribute to reduce the independent particle polarizability even at the ALDA level (this contribution for extended systems is zero and as the long range behavior of the exchange-correlation (XC) potential is not relevant in this regime, this clearly points that the nonlocality of the $\mathrm{XC}$ kernel as well as self-interaction correction are responsible for the bad performance of LDA). We will observe this overestimation in the calculations that follow. In fact, in the case of compact finite systems, there has been indications that the exchange-correlation potential seems to be more important than the kernel. ${ }^{28}$ The situation is particularly problematic in the case of long molecular chains, ${ }^{27}$ where standard exchange-correlation functionals can greatly overestimate polarizabilities when compared to many-body approaches. Note, however, that this is not a deficiency of DFT, but of the LDA approximation (and of many exchange-correlation functionals), that can, in principle, be treated ${ }^{29}$ by using more sophisticated orbital dependent functionals such as the selfinteraction corrected LDA (Ref. 30) or the exact exchange. ${ }^{31}$ However, in the present orbital functionals, there is still a significant correlation contribution that is not taken into account and is responsible for the discrepancies between theory and experiment for long chains in the exact exchange approach. $^{27}$

Numerically, the central part of our scheme is the solution of the Sternheimer equation [Eq. (8)]. This has the form of a linear equation, where the operator to invert is the shifted ground state Hamiltonian. As the shift is complex (due to the $i \eta$ term), this operator is not Hermitian. Therefore, we cannot use standard techniques common in the community, such as the simple conjugated gradient scheme, but have to rely on more general (and involved) linear solvers. Our choice was the biconjugate gradient stabilized method. ${ }^{33}$ Close to the resonance frequencies, the Sternheimer equation becomes very badly conditioned, and the solution process turns out to be very costly. The problem can be eased by the use of preconditioning. We have found that a smoothing preconditioner ${ }^{34}$ can dramatically improve the convergence in these cases. Also for a small system the solution process can be made less costly if we solve the Sternheimer equation in the space of the unoccupied wave functions by orthogonalizing the right-hand side of the Sternheimer equation with respect to the occupied wave functions, although this would not be practical for large systems as the orthogonalization 
TABLE I. Comparison of static polarizabilities and hyperpolarizabilities for CO. Results are in a.u.

\begin{tabular}{lcccccc}
\hline \hline & This work & LDA & HF $^{\mathrm{a}}$ & $\mathrm{MP}^{\mathrm{a}}$ & $\left.\operatorname{CCSD}^{\mathrm{T}}\right)^{\mathrm{a}}$ & Expt. \\
\hline$\mu$ & 0.0631 & & -0.1052 & 0.0905 & 0.057 & $0.0481^{\mathrm{b}}$ \\
$\alpha_{x x}$ & 12.55 & & 11.25 & 12.00 & 11.97 & \\
$\alpha_{z z}$ & 15.82 & & 14.42 & 15.53 & 15.63 & \\
$\bar{\alpha}$ & 13.64 & $13.87^{\mathrm{c}}$ & 12.31 & 13.18 & 13.19 & $13.09^{\mathrm{d}}$ \\
$\beta_{x x z}$ & 8.35 & $8.24^{\mathrm{e}}$ & 5.0 & 8.3 & 8.4 & \\
$\beta_{z z z}$ & 33.34 & $33.52^{\mathrm{e}}$ & 31.1 & 28.3 & 30.0 & \\
$\beta_{\|}$ & 30.03 & $30.00^{\mathrm{e}}$ & 24.8 & 27.0 & 28.0 & \\
\hline \hline
\end{tabular}

${ }^{\mathrm{a}}$ Finite difference results from Ref. 44.

${ }^{\mathrm{b}}$ Experimental result from Ref. 45.

${ }^{c}$ LDA basis set results from Ref. 46.

${ }^{\mathrm{d}}$ Experimental result from Ref. 47.

${ }^{e}$ LDA basis set results from Ref. 48.

process can become very demanding. Even with these techniques, the process is much more costly for frequencies near resonance. For example, for the $\mathrm{CO}$ case, the full selfconsistent solution of the Sternheimer equation for a single frequency in a single direction requires around 1700 applications of the Hamiltonian; for the near resonance frequency, approximately ten times more applications are required. As a comparison, for the ground state DFT calculation, around 2000 Hamiltonian operations are needed.

As the right-hand side of the Sternheimer equation depends on the linear variation of the density, the problem has to be solved self-consistently. For this, we use similar strategies as for the ground state calculation, mixing the linear variation of the density using a Broyden scheme ${ }^{35}$ in order to speed up the convergence of the self-consistent cycle.

The Poisson equation is solved using the interpolating scaling function scheme proposed in Ref. 36. As this process has to be done only once per self-consistency iteration, the performance of the Poisson solver is not critical.

The total cost of calculating the response is of order $\mathcal{O}\left(N_{\mathrm{KS}} N_{g} M_{\omega}\right)$, where $N_{\mathrm{KS}}$ is the number of Kohn-Sham orbitals, $N_{g}$ is the number of grid points, and $M_{\omega}$ is the number of frequencies we desire (which is independent of the system size). This scaling is much better than for the approaches that rely on expansions in particle-hole states, and even better than for the ground state calculation that normally scales as $\mathcal{O}\left(N_{\mathrm{KS}} N_{g}^{2}\right)$ (due to the necessity of orthogonalizing the wave functions). We believe, therefore, that this method can be used to study (hyper)polarizabilities of very large systems, such as nanostructures or molecules with biological interest. After obtaining the linear response, the evaluation of the hyperpolarizability from Eq. (16) has a cost proportional to $\mathcal{O}\left(N_{\mathrm{KS}}^{2} N_{g} M_{\omega}\right)$ but with a very small prefactor. Note that $\mathcal{O}(N)$ (with $N$ the number of atoms) schemes are available for the ground state ${ }^{37}$ and static polarizability calculations. ${ }^{38}$ These linear scaling methods are based on "near sightness"39 or the idea of divide and conquer. ${ }^{40}$ The real-space treatment used in our method would allow for the incorporation of these strategies to reduce even further the numerical cost for large systems.

\section{EXAMPLES OF APPLICATIONS}

We decided to illustrate the implementation of our method by applying it to some simple molecules that have been well studied both experimentally and theoretically: CO,
$\mathrm{H}_{2} \mathrm{O}$, and para-nitroaniline (PNA).

In all our calculations we took the experimental geometries $\overline{\mathrm{CO}}=1.13 \AA, \overline{\mathrm{HO}}=0.957 \AA$, and $\mathrm{HO} \mathrm{H}=104.5^{\circ}$; the experimental geometry of PNA determined through x-ray crystallography can be found in Ref. 41. However, there are two $\mathrm{CH}$ distances missing from the crystallographic data. For these we followed Ref. 42 and took their theoretical values (see Table 1 of Ref. 43). In all cases the dipole moment of the molecule was taken perpendicular to the $z$ axis, for $\mathrm{H}_{2} \mathrm{O}$ the molecule was considered in the $y z$ plane, and in the case of PNA it was taken in the $x z$ plane. We used TroullierMartins norm-conserving pseudopotentials with core radii of $0.66 \AA$ for $\mathrm{H}, 0.78 \AA$ for $\mathrm{C}$, and $0.74 \AA$ for both $\mathrm{N}$ and $\mathrm{O}$.

We used a simulation box composed of spheres centered at each atomic position, with radii of $9.5 \AA$ for $\mathrm{CO}, 7.4 \AA$ for $\mathrm{H}_{2} \mathrm{O}$, and $5.3 \AA$ for PNA. The points were distributed in a regular grid with spacings of $0.20 \AA$ for $\mathrm{CO}, 0.17 \AA$ for $\mathrm{H}_{2} \mathrm{O}$, and $0.19 \AA$ for PNA. With these parameters, hyperpolarizabilities are converged to better than $1 \%$. As expected, the simulation boxes required to converge the hyperpolarizabilities were much larger than the ones typically used in ground state calculations, as these quantities have sizable contributions from the regions far away from the nuclei. The

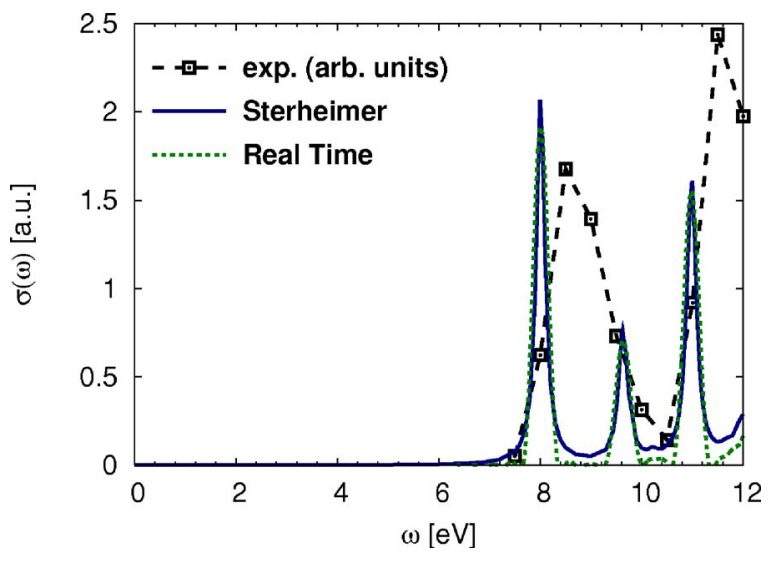

FIG. 1. Average photoabsorption cross section of the $\mathrm{CO}$ molecule, calculated within the adiabatic LDA. The line corresponds to the results obtained through the solution of the Sternheimer equation, while the dots are obtained through the solution in real time of the time-dependent Kohn-Sham equation. Low resolution experimental absorption cross section from Ref. 49. For a more detailed comparison, the relevant experimental excitation energies are at $8.51 \mathrm{eV}\left(A^{1} \Pi\right), 10.78 \mathrm{eV}\left(B^{1} \Sigma^{+}\right), 11.40 \mathrm{eV}\left(C^{1} \Sigma^{+}\right)$, and $11.53 \mathrm{eV}$ $\left(E^{1} \Pi\right)$ (Refs. 49 and 50). 


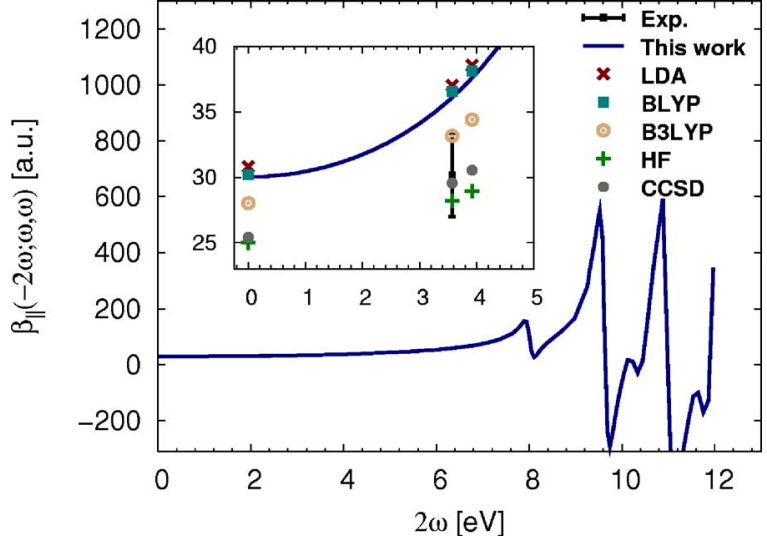

FIG. 2. (Color online) Second harmonic generation $\beta_{\|}(-2 \omega ; \omega, \omega)$ of CO. The inset shows a comparison of the results of this work (TDLDA) with other available results. Exp., experimental results from Ref. 5; HF, HartreeFock results from Ref. 42; LDA, BLYP, and B3LYP, DFT results from Ref. 42; and CCSD, coupled cluster results from Ref. 42.

required simulation box size depends on the frequency of the perturbation, being larger for higher frequencies. Finally, we used the LDA parametrization by Perdew and Zunger ${ }^{30}$ to approximate the exchange-correlation functionals.

We will start our discussion with CO. The results for static properties are displayed in Table I. The results fully agree with the other DFT-LDA calculations. Compared to more sophisticated quantum-chemistry methods, the LDA overestimates the values for the polarizabilities and the hyperpolarizabilities. This, as mentioned before, comes from a deficiency in the asymptotic region of the LDA potential.

We now turn to the dynamic properties. In Fig. 1 we plot the absorption cross section of the $\mathrm{CO}$ molecule obtained with our approach and compare to the spectrum obtained through the direct solution of the time-dependent KohnSham equations in real time. As expected, the two calculations agree perfectly, which validates our numerical implementation of the Sternheimer equations. Note that, even if both methods have identical scalings, the solution of the Sternheimer equation still has a larger prefactor if the whole spectrum is required. This is due to the ill conditioning of the linear system close to resonances. From Fig. 1 it is clear that the two theoretical results are redshifted with respect to the experimental curve. This shortcoming of the simple LDA can be corrected by using functionals with the correct asymptotic behavior. $^{29}$

In Fig. 2 we present our calculations of the second har-
TABLE III. Comparison of (hyper)polarizabilities of $\mathrm{H}_{2} \mathrm{O}$ for different DFT calculations. $\operatorname{CCSD}(\mathrm{T})$ and experimental values are given as references. For dynamic results $\omega=1.79 \mathrm{eV}$.

\begin{tabular}{|c|c|c|c|c|}
\hline & $\alpha(0,0)$ & $\beta_{\|}(0 ; 0,0)$ & $\beta_{\|}(0 ; \omega,-\omega)$ & $\beta_{\|}(-2 \omega ; \omega, \omega)$ \\
\hline This work (TDLA) & 10.51 & -25.89 & -28.33 & -34.71 \\
\hline This work (KLI/ALDA) & 8.61 & -11.75 & -12.43 & -14.03 \\
\hline $\mathrm{LDA}^{\mathrm{a}}$ & 10.5 & -26.1 & -28.6 & -35.1 \\
\hline BLLYP $^{a}$ & 10.8 & -27.9 & -30.9 & -38.8 \\
\hline $\mathrm{LB}^{\mathrm{a}}{ }^{\mathrm{a}}$ & 9.64 & -17.8 & -17.7 & -20.3 \\
\hline $\mathrm{LDA}^{\mathrm{b}}$ & 10.63 & -23.78 & -26.09 & -32.12 \\
\hline BLYP $^{b}$ & 10.77 & -23.65 & -26.11 & -32.76 \\
\hline B3LYP ${ }^{b}$ & 9.81 & -18.54 & -20.11 & -24.11 \\
\hline $\mathrm{HF}^{\mathrm{b}}$ & 8.53 & -10.73 & -11.27 & -12.52 \\
\hline $\operatorname{CCSD}(T)^{\mathrm{c}}$ & 9.79 & -18.0 & -19.0 & -21.1 \\
\hline Expt. & $9.81^{\mathrm{d}}$ & & & $-22 \pm 9^{e}$ \\
\hline
\end{tabular}

${ }^{\mathrm{a}}$ Grid based calculations from Ref. 17.

${ }^{\mathrm{b}}$ Basis set calculations from Ref. 42.

${ }^{\mathrm{c}}$ Results from Ref. 54.

${ }^{\mathrm{d}}$ Experimental results from Ref. 55.

${ }^{\mathrm{e}}$ Experimental results from Ref. 56.

monic generation spectrum $\beta_{\|}(-2 \omega ; \omega, \omega)$ of $\mathrm{CO}$, together with the available experimental results ${ }^{5}$ and previous theoretical data. ${ }^{42}$ Our results agree very well with previous DFT results using the LDA. We can also see that the use of the generalized gradient approximation BLYP [Becke 88 (Ref. 51) for exchange and Lee-Yang-Parr ${ }^{52}$ correlation] does not change significantly the results. Using a hybrid functional, the Becke 3 parameter B3LYP functional ${ }^{53}$ does reduce the error, while Hartree-Fock (HF) results underestimate the value for the hyperpolarizability. The best results are, as expected, obtained by coupled cluster calculations using singles and doubles (CCSD).

Now we turn to the $\mathrm{H}_{2} \mathrm{O}$ molecule. To test our implementation, we show, in Table II, the different components of the hyperpolarizability tensor for $\omega=0,1.79$, and $1.96 \mathrm{eV}$. We see that our results compare well to previous theoretical work. ${ }^{42}$ The small difference can be explained by the different numerical methodologies (real-space grid and pseudopotentials in our case and basis sets in Ref. 42).

In Table III we show the static polarizability $\alpha(0,0)$, the static first hyperpolarizability $\beta_{\|}(0 ; 0,0)$, the optical rectification $\beta_{\|}(0 ; \omega,-\omega)$, and second harmonic generation $\beta_{\|}(-2 \omega ; \omega, \omega)$ for water. All dynamic values were calculated for $\omega=1.79 \mathrm{eV}$. This time we also have included results with the KLI orbital dependent exchange-correlation potential

TABLE II. Tensor components for the second harmonic generation $\beta(-2 \omega ; \omega, \omega)$ of $\mathrm{H}_{2} \mathrm{O}$.

\begin{tabular}{|c|c|c|c|c|c|c|}
\hline & \multicolumn{2}{|c|}{$\omega=0.00 \mathrm{eV}$} & \multicolumn{2}{|c|}{$\omega=1.79 \mathrm{eV}$} & \multicolumn{2}{|c|}{$\omega=1.96 \mathrm{eV}$} \\
\hline & This work & $\mathrm{LDA}^{\mathrm{a}}$ & This work & $\mathrm{LDA}^{\mathrm{a}}$ & This work & $\mathrm{LDA}^{\mathrm{a}}$ \\
\hline$\beta_{z z z}$ & -21.23 & -19.14 & -27.67 & -25.22 & -29.37 & -26.81 \\
\hline$\beta_{z x x}$ & -9.84 & -8.82 & -12.89 & -11.45 & -13.68 & -12.11 \\
\hline$\beta_{x x z}$ & -9.84 & -8.82 & -16.87 & -15.57 & -19.28 & -17.90 \\
\hline$\beta_{z y y}$ & -12.08 & -11.67 & -14.94 & -14.39 & -15.68 & -15.09 \\
\hline$\beta_{y y z}$ & -12.08 & -11.67 & -14.48 & -13.99 & -15.06 & -14.56 \\
\hline
\end{tabular}

${ }^{\mathrm{a} T h e o r e t i c a l ~ r e s u l t s ~ f r o m ~ R e f . ~} 42$. 


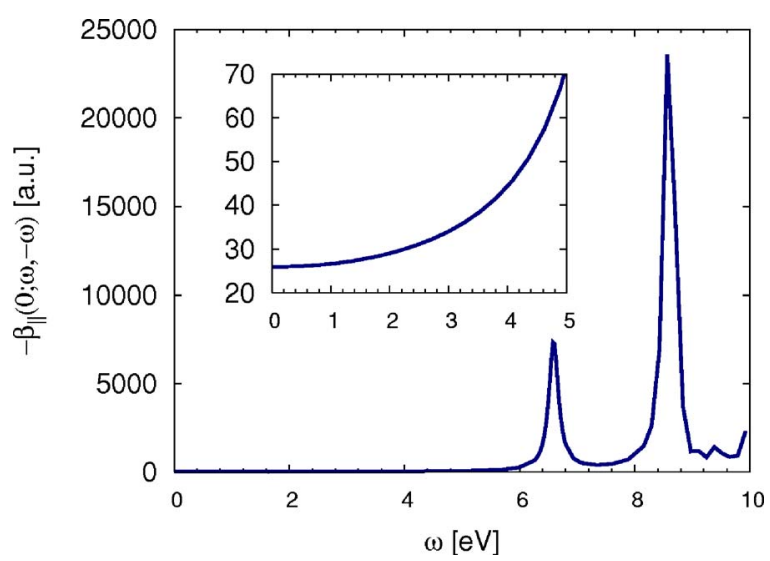

FIG. 3. Calculated optical rectification $\beta_{\|}(0 ; \omega ;-\omega)$ of $\mathrm{H}_{2} \mathrm{O}$.

combined with the ALDA exchange-correlation kernel. Concerning LDA values, we can see that our results fully agree with the results of Ref. 17 that also uses a grid based representation, while basis set results from Ref. 42 differ by less than $10 \%$. We can see that all LDA and BLYP values overestimate the magnitude of (hyper)polarizabilities with respect to experiment and coupled cluster calculations. The use of more sophisticated exchange-correlation functionals, such as LB94 or B3LYP, improves significantly the results, while the KLI/ALDA scheme gives an underestimation of the magnitude of (hyper)polarizabilities similar to Hartree-Fock results.

To conclude the discussion of water results, we plot in Fig. 3 the frequency dependence of the optical rectification in the visible and near ultraviolet regimes. It is clear that our method not only works for small nonresonant frequencies but also in the more complicated resonant regime.

Finally, we turn to a larger molecule: para-nitroaniline. Our results for the second harmonic generation process in this molecule are given in Fig. 4. There are two experiments available: (i) a gas phase experiment ${ }^{57}$ performed for a single frequency and (ii) para-nitroaniline in solvent for several frequencies. ${ }^{58}$ The latter was corrected for the presence of the solvent, but this correction is clearly incomplete as the value from Ref. 58 for $\omega=1.17 \mathrm{eV}$ is still $10 \%$ larger that the gas phase measurement. ${ }^{57}$ We include also other theoretical values using DFT (Refs. 48 and 59) and CCSD. ${ }^{42}$ We can observe that our results underestimate the solvent experimental results by about $15 \%$ for all available frequencies. In comparison, B3LYP and CCSD results are seriously too small at high frequencies, with values that are around $40 \%-$ $50 \%$ smaller than experiment. We think that the reason for this discrepancy is the better description of the hyperpolarizabilities near resonance of our method. Furthermore, it uses a grid based representation that describes better the regions far from the nuclei in comparison with localized basis sets, allowing for larger flexibility in capturing the dynamic changes in the wave functions.

\section{CONCLUSIONS}

In summary, we have presented a method that allows the calculation of both static and dynamic polarizabilities and hyperpolarizabilities. Our approach is based on the Sternheimer equation, within the formalism of time-dependent density functional theory, and requires the solution of a nonHermitian linear equation. This solution is obtained through a generalization of the conjugated gradient method using a real-space (basis-set-free) representation of the wave functions. In this way we are able to obtain not only static quantities but also the whole frequency dependence of the (hyper)polarizabilities even close to resonances. The scaling with the number of atoms in the system is excellent, so we expect that the method will be useful for the study of very large systems. First applications to small benchmark molecules yield quite good results in comparison with previous theoretical approaches and experimental results.

One of the beauties of this approach is how easily it can be generalized to higher orders and to handle other kinds of static or dynamic perturbations. For example, phonon frequencies, (resonant) Raman tensors, NMR tensors, forces in the excited state, etc., can all be obtained by just changing the right-hand side of the Sternheimer equations. The third and higher order polarizabilities can also be obtained by solving a hierarchy of Sternheimer equations that have the
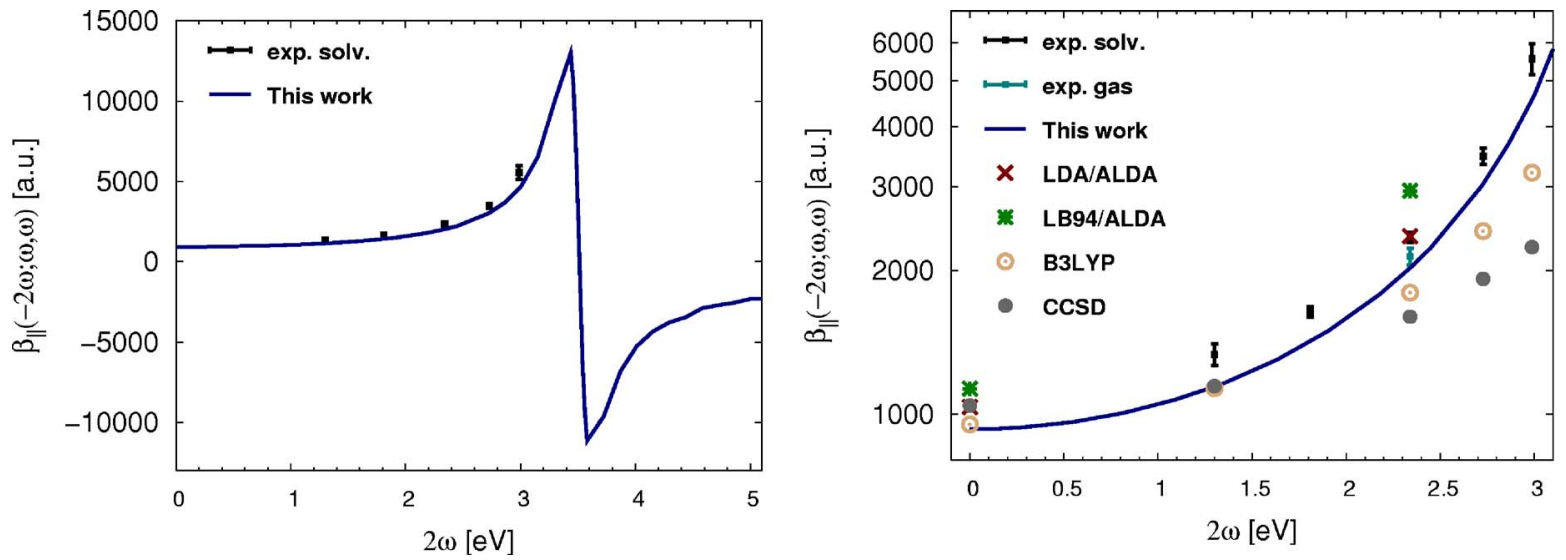

FIG. 4. (Color online) Second harmonic generation $\beta_{\|}(-2 \omega ; \omega, \omega)$ of para-nitroaniline. Note that the $y$ axis in the right panel is in logarithmic scale. exp. solv., solvent phase experimental results from Ref. 58; exp. gas, gas phase experimental results from Ref. 57; LDA/ALDA and LB94/ALDA, DFT basis set results from Ref. 48; B3LYP, DFT basis set results from Ref. 59; and CCSD, coupled cluster results from Ref. 42. Some references use a different convention to define hyperpolarizabilities; all the values shown here have been converted to convention $A B$. 
same form as the first order one. Work has already started to extend our implementation in these directions.

\section{ACKNOWLEDGMENTS}

The authors were partially supported by the EC Network of Excellence NANOQUANTA (NMP4-CT-2004-500198), SANES Project (NMP4-CT-2006-017310), DNANANODEVICES (IST-2006-029192), NANO-ERA Chemistry, MCyT, and Barcelona Supercomputing Center (Mare Nostrum). One of the authors (X.A.) would also like to acknowledge partial support from the EU Programme Marie Curie Host Fellowship (HPMT-CT-2001-00368).

${ }^{1}$ P. N. Butcher and D. Cotter, The Elements of Nonlinear Optics (Cambridge University Press, Cambridge, 1990).

${ }^{2}$ N. Bloembergen, Nonlinear Optics (Benjamin, New York, 1965).

${ }^{3}$ K. D. Bonin and V. V. Kresinq, Electric-Dipole Polarizabilities of Atoms, Molecules and Clusters (World Scientific, Singapore, 1997).

${ }^{4}$ A. Dalgarno, in Perturbation Theory and Its Applications in Quantum Mechanics, edited by C. H. Wilcox (Wiley, New York, 1996), p. 162; P. W. Langhoff, S. T. Epstein, and M. Karplusaroni, Rev. Mod. Phys. 44, 602 (1972).

${ }^{5}$ D. P. Shelton and J. E. Rice, Chem. Rev. (Washington, D.C.) 94, 3 (1994).

${ }^{6}$ D. M. Bishop and P. Norman, in Handbook of Advanced Electric and Photonic Materials and Devices: Nonlinear Optical Materials, edited by H. S. Nalwa (Academic, San Diego, 2000), Vol. 9, Chap. 1.

${ }^{7}$ S. Baroni, S. de Gironcoli, A. D. Corso, and P. Gianozzi, Rev. Mod. Phys. 73, 515 (2001), and references therein.

${ }^{8}$ C. Cardoso, F. Nogueira, and M. A. L. Marques (unpublished).

${ }^{9}$ See, for example, J. E. Gready, G. B. Backsay, and N. S. Hush, Chem. Phys. 22, 141 (1977), this method is widely used in the chemistry and physics communities.

${ }^{10}$ B. J. Orr and J. F. Ward, Mol. Phys. 20, 513 (1971).

${ }^{11}$ A. Zangwill, J. Chem. Phys. 78, 5926 (1983); K. R. Subbaswamy and G. D. Mahan, ibid. 84, 3317 (1986); G. Senatore and K. R. Subbaswamy, Phys. Rev. A 34, 3619 (1986).

${ }^{12}$ K. Yabana and G. F. Bertsch, Phys. Rev. B 54, 4484 (1996).

${ }^{13}$ R. Sternheimer, Phys. Rev. 84, 244 (1951).

${ }^{14}$ G. Senatore and K. R. Subbaswamy, Phys. Rev. A 35, 2440 (1987)

${ }^{15}$ S. P. Karna and M. Dupuis, Chem. Phys. Lett. 171, 201 (1990).

${ }^{16}$ S. J. A. van Gisbergen, J. G. Snijders, and E. J. Baerends, Phys. Rev. Lett. 78, 3097 (1997).

${ }^{17}$ J.-I. Iwata, K. Yabana, and G. F. Bertsch, J. Chem. Phys. 115, 8773 (2001).

${ }^{18}$ B. Walker, A. M. Saitta, R. Gebauer, and S. Baroni, Phys. Rev. Lett. 96, 113001 (2006).

${ }^{19}$ P. Hohenberg and W. Kohn, Phys. Rev. 136, B864 (1964); W. Kohn and L. J. Sham, ibid. 140, A1133 (1965).

${ }^{20}$ E. Runge and E. K. U. Gross, Phys. Rev. Lett. 52, 997 (1984); TimeDependent Density Functional Theory, edited by M. A. L. Marques, C. A. Ullrich, F. Nogueira, A. Rubio, K. Burke, and E. K. U. Gross (Springer, Heidelberg, 2006).

${ }^{21}$ A. D. Corso, F. Mauri, and A. Rubio, Phys. Rev. B 53, 15638 (1996).

${ }^{22}$ X. Gonze, Phys. Rev. A 52, 1086 (1995).

${ }^{23}$ A. Willetts, J. E. Rice, D. M. Burland, and D. P. Shelton, J. Chem. Phys. 97, 7590 (1992).

${ }^{24}$ X. Gonze and J.-P. Vigneron, Phys. Rev. B 39, 13120 (1989).

${ }^{25}$ M. A. L. Marques, A. Castro, G. F. Bertsch, and A. Rubio, Comput. Phys. Commun. 151, 60 (2003); A. Castro, H. Appel, M. Oliveira, C. A. Rozzi, X. Andrade, F. Lorenzen, M. A. L. Marques, E. K. U. Gross, and A. Rubio, Phys. Status Solidi B 243, 2465 (2006).
${ }^{26}$ G. E. Scuseria and V. N. Staroverov, in Theory and Applications of Computational Chemistry: The First Forty Years, edited by C. E. Dykstra, G. Frenking, K. S. Kim, and G. E. Scuseria (Elsevier, Amsterdam, 2005), pp. $669-724$

${ }^{27}$ S. van Gisbergen, P. Schipper, O. Gritsenko, E. Baerends, J. Snijders, B. Champagne, and B. Kirtman, Phys. Rev. Lett. 83, 694 (1999); P. de Boeij, F. Kootstra, J. Berger, R. van Leeuwen, and J. Snijders, J. Chem. Phys. 115, 1995 (2001); M. van Faassen, P. de Boeij, F. Kootstra, J. Berger, R. van Leeuwen, and J. Snijders, ibid. 118, 1044 (2003); P. Mori-Sánchez, Q. Wu, and W. Yang, ibid. 119, 11001 (2003); S. Kümmel, L. Kronik, and J. P. Perdew, Phys. Rev. Lett. 93, 213002 (2004).

${ }^{28}$ M. Stener, P. Decleva, and A. Grling, J. Chem. Phys. 114, 7816 (2001).

${ }^{29}$ M. E. Casida and D. R. Salahub, J. Chem. Phys. 113, 8918 (2000); M. A. L. Marques, A. Castro, and A. Rubio, ibid. 115, 3006 (2001); F. A. Bulat, A. Toro-Labbé, B. Champagne, B. Kirtman, and W. Yang, ibid. 123, 014319 (2005).

${ }^{30}$ J. P. Perdew and A. Zunger, Phys. Rev. B 23, 5048 (1981).

${ }^{31}$ A. Görling and M. Levy, Phys. Rev. A 50, 196 (1994).

${ }^{32}$ J. B. Krieger, Y. Li, and G. J. Iafrate, Phys. Lett. A 146, 256 (1990).

${ }^{33}$ Y. Saad, Iterative Method for Sparse Linear Systems (Pws, Boston, 1996).

${ }^{34}$ Y. Saad, A. Stathopoulos, J. Chelikowsky, K. Wu, and S. Öğüt, BIT 36, 563 (1996).

${ }^{35}$ C. G. Broyden, Math. Comput. 19, 577 (1965); D. D. Johnson, Phys. Rev. B 38, 12807 (1988).

${ }^{36}$ L. Genovese, T. Deutsch, A. Neelov, S. Goedecker, and G. Beylkin, J. Chem. Phys. 125, 074105 (2006), we use the free implementation of this solver provided by the authors.

${ }^{37}$ S. Goedecker, Rev. Mod. Phys. 71, 1085 (1999).

${ }^{38}$ H. J. Xiang, J. Yang, J. G. Hou, and Q. Zhu, Phys. Rev. Lett. 97, 266402 (2006).

${ }^{39}$ W. Kohn, Phys. Rev. Lett. 76, 3168 (1996).

${ }^{40}$ W. Yang, Phys. Rev. Lett. 66, 1438 (1991).

${ }^{41}$ F. Bertinelli, P. Palmieri, A. Brillante, and C. Taliani, Chem. Phys. 25, 333 (1977).

${ }^{42}$ P. Salek, T. Helgaker, O. Vahtras, H. Ågren, D. Jonsson, and J. Gauss, Mol. Phys. 103, 439 (2005).

${ }^{43}$ S. P. Karna, P. N. Prasad, and M. Dupuis, J. Chem. Phys. 94, 1171 (1991).

${ }^{44}$ G. Maroulis, J. Phys. Chem. 100, 13466 (1996).

${ }^{45}$ J. S. Muenter, J. Mol. Spectrosc. 55, 490 (1975).

${ }^{46}$ S. J. A. van Gisbergen, F. Kootstra, P. R. T. Schipper, O. V. Gritsenko, J. G. Snijders, and E. J. Baerends, Phys. Rev. A 57, 2556 (1998).

${ }^{47}$ G. A. Parker and R. T. Pack, J. Chem. Phys. 64, 2010 (1976).

${ }^{48}$ S. J. A. van Gisbergen, J. G. Snijders, and E. J. Baerends, J. Chem. Phys. 109, 10644 (1998); 111, 6652 (E) (1999).

${ }^{49}$ W. Chan, G. Cooper, and C. Brion, Chem. Phys. 170, 123 (1993).

${ }^{50}$ E. S. Nielsen, P. Jørgensen, and J. Oddershede, J. Chem. Phys. 73, 6238 (1980); 75, 499 (E) (1981)

${ }^{51}$ A. Becke, Phys. Rev. A 38, 3098 (1988).

${ }^{52}$ C. Lee, W. Yang, and R. Parr, Phys. Rev. B 37, 785 (1988); B. Miehlich, A. Savin, H. Stoll, and H. Preuss, Chem. Phys. Lett. 157, 200 (1989).

${ }^{53}$ A. D. Becke, J. Chem. Phys. 98, 5648 (1993).

${ }^{54}$ H. Sekino and R. Bartlett, J. Chem. Phys. 98, 3022 (1993).

${ }^{55}$ M. A. Spackman, J. Phys. Chem. 93, 7594 (1989).

${ }^{56}$ J. F. Ward and C. K. Miller, Phys. Rev. A 19, 826 (1979).

${ }^{57}$ P. Kaatz, E. A. Donley, and D. P. Shelton, J. Chem. Phys. 108, 849 (1998).

${ }^{58}$ C. C. Teng and A. F. Garito, Phys. Rev. B 28, 6766 (1983).

${ }^{59}$ P. Salek, O. Vahtras, T. Helgaker, and H. Ågren, J. Chem. Phys. 117, 9630 (2002)

${ }^{60}$ This is straightforward to prove by expanding the variation of the wave functions in terms of the ground state wave functions, using the standard perturbation theory, and then replacing the resulting expression in the variation of the density [Eq. (7)]. 